\title{
Correlation of brain-dead organ donors' age and time period between admission and the first brain death examination: 5 years data of Korea
}

\author{
Eun-sil Jeong ${ }^{1}$, A. S. M. Tanim Anwar' ${ }^{2}$, Jae-myeong Lee ${ }^{1}$ \\ ${ }^{1}$ Transplantation Center, Korea University Anam Hospital, Seoul, Korea \\ ${ }^{2}$ Department of Nephrology, Dhaka Medical College Hospital, Dhaka, Bangladesh
}

Background: Deceased organ donation can be performed only with the consent of the donor or their family members. We tried to identify whether donor age is related to decision making for the families to give consent for organ donation.

Methods: In this study, we reviewed data of 2,451 brain-dead organ donors (male, 1,645; female, 806; mean age, 46.5 \pm 16.2 years) from Korean Network for Organ Sharing (KONOS) registry pertaining to the period between December 2012 and December 2017. We assessed the time period between the admission of the patient and the first brain death assessment.

Results: We found that the mean duration from the admission to the first brain death examination was significantly longer in the age group of $0-30$ years ( $16.23 \pm 6.01$ days), compared to age group $31-83$ years $(6.7 \pm 1.07$ days; $P=0.001)$. There was a strong negative correlation $(r=0.795, P=0.010)$ between age and the mean duration from the admission to the first brain death examination.

Conclusions: As the family members of the younger brain-dead donors needed more time to consent for organ donation compared to those of older donors, the first brain death examination and donation process delayed in case of young donors. 